\title{
Networked Virtual Organizations: A Chance for Small and Medium Sized Enterprises on Global Markets
}

\author{
Wojciech Cellary* \\ Poznan University of Economics \\ Department of Information Technology \\ Mansfelda 4, 60-854 Poznan, Poland \\ cellary@kti.ue.poznan.pl
}

\begin{abstract}
Networked Virtual Organizations (NVOs) are a right answer to challenges of globalized, diversified, and dynamic contemporary economy. NVOs need more than e-trade and outsourcing, namely, they need out-tasking and e-collaboration. To out-task, but retain control on the way a task is performed by an external partner, two integrations are required: (1) integration of computer management systems of enterprises cooperating within an NVO; and (2) integration of cooperating representatives of NVO member enterprises into a virtual team. NVOs provide a particular chance to Small and Medium size Enterprises (SMEs) to find their place on global markets and to play a significant role on them. Requirements for SMEs to be able to successfully join an NVO are analyzed in the paper.
\end{abstract}

Keywords: virtual organizations, small and medium size enterprises, core competences, context competences, virtual teams.

\section{Introduction}

Computer science and engineering has been developing faster than any other discipline in the history of human civilization. Also, current spread of computer applications has no precedence [6]. Computer science revolutionized business management providing a possibility to store arbitrarily detailed information about an enterprise and every kind of its activity in databases, as well as providing a possibility to process this information for managerial reasons. Due to computer science and engineering, it was possible to abandon uniformization of business processes and to shift to mass personalization on a global scale. As a consequence, it was possible to better serve diversified clients, and better exploit business opportunities arising on dynamically changing markets.

Development and mass deployment of information and communication technologies followed by organizational changes of businesses, as well as increased customer

\footnotetext{
* This work has been partially supported by the Polish Ministry of Science and Higher Education within the European Regional Development Fund, Grant No. POIG.01.03.01-00-008/08. 
expectations and market globalization challenge enterprises. This challenge is particularly important for Small and Medium sized Enterprises (SMEs) that up to date worked mostly at local markets in relatively stable environments [5].

In this paper we argue that to face the challenges of today economy, Networked Virtual Organizations are the proper business structure. Big, hierarchical corporations should flatten to become Networked Virtual Organizations, while SMEs should integrate in Networked Virtual Organizations.

In Section 2 we briefly characterize contemporary business environment showing the impact of information and communication deployment on businesses. In Section 3 we present the concept of networked virtual organizations. Section 4 is devoted to features of networked virtual organizations, while Section 5 to the requirements an SME has to satisfy to be able to participate in an NVO. In Section 6 two integrations are discussed: that of computer management systems and that of representatives of NVO member enterprises into virtual teams. In Section 7 ongoing research on Virtual Breeding Environments, as an intermediary between a universe of enterprises and NVOs, is described. Finally, Section 8 concludes the paper.

\section{Business Environment}

Due to features of electronic information and communication, contemporary enterprises and their employees are available all the time in a space without geographical constraints. Computers make it possible to acquire information from everywhere in real time and to contact anybody in every moment. Of course, words: "everywhere", "everybody", and "any moment" concern mostly the advanced part of the world and advanced parts of developing countries, but this part of the world sets the ton of global economy.

Constant availability in a space without geographical constraints is followed by dynamism and diversity of business activities. Contemporary economy is characterized by permanent changes. Markets are changing, both from the macroeconomic and microeconomic perspectives. From the macroeconomic perspective - because competitive conditions are changing. From the microeconomic perspective - because enterprises are permanently trying to enter new markets with innovative products and services. Customers, suppliers and business partners of an enterprise are changing, because finding and contacting them is easy due to electronic communication. Technologies of production and service delivery are changing due to innovations following from research and development. Innovation is considered to be the main driver of competitiveness. Innovations are spreading faster than ever again due to electronic information and communication. Many changes are following from permanent improvement of computer software used in production and service delivery. Also methods of work are changing, which requires news skills from employees. These new skills are mostly related to the use of software supporting employees in their work. Organization of work is changing as well, in particular workplaces based on information and communication. A good example of organizational changes is teleworking. Finally, technological and organizational changes are followed by indispensable changes of law. 
Differentiation of enterprise activities may be perceived on both macro- and microeconomic level. On the macroeconomic level, increasing number of enterprises, including SMEs, are present on global markets. They have to deal with different economical conditions and law systems in different countries. Even in such integrated multinational organizations as European Union, law requirements and administrative procedures in different countries are far not the same. This requires from enterprises a lot of specific knowledge. More subtle and therefore more difficult to manage are cultural differences. Knowledge of cultural nuances is often a necessary condition for business success. Culture is often a determining factor for acceptance or nonacceptance of a given product or service on a particular market.

On the microeconomic scale, a contemporary tendency is realization of holistic needs of a customer. From an enterprise point of view, a motivation for such approach is conviction that the most costly and difficult part of any business process is, first, to win a customer, and then to retain him/her. Therefore, a won customer has to be provided with the largest possible offer of complementary products and services. This means that an enterprise needs to have a wide offer of such products and services at customer's disposal.

\section{Concept of Networked Virtual Organizations}

A right answer to challenges of globalized, diversified, and dynamic business environment presented in Section 2 are Networked Virtual Organizations. A Networked Virtual Organization (NVO) is a set of business and/or administrative units mutually cooperating through a computer network, perceived on the market as if they were a single enterprise [1], [3], [4].

There are two possible processes of transforming enterprises to NVOs: decomposition of traditional, hierarchical organizations, and integration of small and medium sized enterprises. In the paper era, when information and documents flow was slow, costly and geographically dependent, hierarchical organizations were efficient. To minimize costs of information flow, it was reasonable and justified to assume rigid roles and functions of organization component units, as well as their hierarchical structure. When rigid roles of units were assumed, the units knew what and when to do even in case of reduced, costly and inefficient communication. It is worth to add that not long ago, when the use of computers in the production processes and the amount of computer supported services was low, the enterprises were not able to rapidly change the production and provided services. Therefore, the market was not dynamic, so also the need for fast communication was not so important.

In contemporary economy, where electronic information and communication is dominating (though often replicated on paper) the hierarchical organization based on fixed functions and roles is not efficient any more. In the electronic information era, business decisions should be based on instant communication instead of rigid rules and functions fixed in advance in the hierarchical organization. Since the employees of any enterprise may capture information all the time, from everywhere, in real time, and contact each other at every moment, their creativity and entrepreneurship should not be limited by the fixed structure of the organization, especially as modern technology offers possibility to rapidly change production and services provided. In the 
electronic information and communication era, a flat network organization provides more opportunities for dynamic business process optimization and better possibilities to adapt businesses to rapid changes on the markets.

A parallel phenomenon to decomposition and flattening of big, hierarchical organizations is integration of SMEs into larger networked virtual organizations. The main driver for SME integration is the conviction that a single, small or medium sized enterprise is not able to face challenges of the dynamic and diversified global markets. Therefore, such an SME should join a bigger economic organization. A selforganization of SMEs into NVOs is a good solution, because it allows SMEs to retain their identity and independence that is very important to SME owners. In the integration process, SMEs can join NVOs coming from decomposition of the big, hierarchical organizations opening their structures for independent SMEs, or self-organize into NVOs in order to offer more complex products and services on the market and to improve their competitive position on the global market.

\section{Features of Networked Virtual Organizations}

There are three main features of NVOs [1]. First, business culture and way of functioning is focused on constantly evolving customer needs. Second, focusing of each NVO component unit on improvement of its own core competences and entrusting of all other functions to partners from the NVO. Third, standardization of data, software systems, and business processes within the whole NVO to achieve higher economic effectiveness.

The first feature meets modern market requirements, where, as mentioned in Section 2, competitiveness is - first of all - achieved through ability to accommodate fast changes. Business culture focused on following the changes on the market demands promotion of creativity and innovation. An enterprise has to be organized in such a way that innovative ideas are driven to deployment.

The second feature of NVO component units - improvement of their own core competences - demands from enterprises to reject the conviction that they are selfsufficient and to entrust non-core functions to partners from the NVO, for which these functions are core competences. Only breaking distrust between partners permits an enterprise to focus on developing and improving its own core competences. A core competence is defined as the one that directly contributes to the competitive advantage of an enterprise on the future market.

To focus on its core competences, an enterprise has to transform its functioning and operation. Functional transformation consists of outsourcing and out-tasking. In case of outsourcing, an enterprise entrusts control over the entire business process to an NVO partner enterprise. The outsourcing enterprise is interested only in the results of the entrusted business process. In case of out-tasking, the enterprise retains control over the way a process is performed by an NVO partner enterprise. Outsourcing of enterprise non-mission critical activities is a method well known and widely used to reduce enterprise operating costs. On the contrary, out-tasking is a relatively new method characteristic for networked virtual organizations. Out-tasking concerns enterprise non core but mission critical activities, such as research and development of 
products and services. This kind of activities demands very close cooperation between partners, under control of the out-tasking enterprise.

The third main feature of NVOs members is standardization of data, software systems and business processes within the whole organization. Standardization is required to provide an NVO with high economic effectiveness. Standardization is far the best method of integration from the operational point of view. It reduces costs, because it eliminates translation of one partner enterprise standard to another, facilitates software systems integration, and enables common realization of complex, computer supported business processes. It should be emphasized that in contemporary economy, a computer system providing tools just to manage internal enterprise resources and processes is not sufficient anymore. An enterprise system must provide external integration, i.e., it must be able to serve and control cross-enterprise processes within an NVO.

Standardization is a key condition enabling fast external integration. Speed of external integration is essential, because a need of immediate cooperation between partners can appear suddenly, as a result of an unexpected business opportunity. Capacity to take up such cooperation depends on the ability of computer systems of business partners to externally integrate.

\section{NVO Requirements for SMEs}

In contemporary economy, small and medium sized enterprises can play an important role, but only under condition that they are able to cooperate within networked virtual organizations. NVOs are required to quickly provide holistic solutions in response to changing customer needs arising in dynamic and diversified environments. This requirement goes beyond traditional expectation that an enterprise possesses competences sufficient to gain competitive advantage in a sector of production or services. Of course, this traditional requirement remains valid, but it is not anymore sufficient to achieve success on the market. A key competence that determines success in modern economy is the ability to cooperate with different business partners via network using information and communication technologies. To develop these competences, an enterprise has to collect and update information resources in formats that are standard within the networked virtual organization. Otherwise, this information is not ready for fast integration and thus useless.

An enterprise has to gain adequate knowledge that is wider than the professional knowledge of its economical sector. Wider knowledge is necessary to correctly interpret information coming from different partners from other parts of the world, other economical sectors, other professions, etc. This information is necessary to discover new business opportunities and to join promising business ventures emerging on evolving markets.

An enterprise has to know about capabilities of its NVO partners. Then, in case of a business opportunity, an enterprise is able to quickly organize an executive team, or at least to notify the NVO about the business opportunity not to be missed.

Employees of an enterprise must be skilled in communication and negotiation via network. Such skills are not very common among SMEs working in traditional sectors of production and services. Without such skills cooperation within an NVO is impossible. 
Further requirements for SMEs to successfully join a networked virtual organization are the following: activity, multiculturalism, interdisciplinarity, creativity and innovation.

Activity is indispensable in a dynamic economy without fixed roles and functions. In such economy, a passive enterprise risks to be excluded from the market.

Multiculturalism is necessary to face challenges of globalization. Ignorance of cultural requirements prevailing on remote markets makes expansion on them impossible. Cooperation within a networked virtual organization with partners from different regions and cultures requires at least basic knowledge of those cultures.

Interdisciplinarity is necessary to meet holistic customer requirements, instead of only partial ones. To face holistic customer needs, professionals from different fields have to cooperate, e.g., economists, lawyers, engineers and artists. Without basic mutual comprehension of other fields, such cooperation is at least difficult, if not impossible.

Finally, creativity and innovativeness are the best strategy for maintaining and improving competitive position on dynamically evolving markets. Creativity and innovativeness can be achieved only in favorable business culture, which is quite rare due to natural conservative attitudes. Four kinds of innovations are distinguished [8].

- Product innovation that means providing the market with a new product or service, or significant improvement of previously offered products or services with reference to their characteristics or destination. Improvement can concern technical characteristics, components, materials, implemented software, more friendly interfaces, or other functional features.

- Process innovation consists of deployment of a new or an improved way a given product or service is produced or delivered.

- Marketing innovation means a new marketing method that encompasses significant changes in product appearance, packaging, positioning, advertising, business model or pricing policy.

- Organizational innovation consists of restructuring an enterprise, or reengineering enterprise business processes, new organization of workplace or external relationships.

The above kinds of innovation cannot be ranked by their importance for an enterprise. Each of them may contribute to improvement of the competitive position of an enterprise or the whole NVO on the market. Therefore, each of them should be carefully developed.

\section{Two Integrations: Computer Management Systems and Virtual Teams}

An SME that wants to be a member of an NVO has to integrate its computer management system with the systems of the remaining NVO members, and to integrate some of its employees with the representatives of NVO member enterprises into a virtual team.

Integration of computer management systems of different enterprises is a costly process. A candidate for an NVO member needs to trust that benefits of the integration 
are higher than costs of integration. The cheaper is the integration, the shorter cooperation within an NVO can be, i.e., more dynamic an NVOs can be built.

There are two main approaches to integration of computer management systems: standardization and intermediation. As mentioned in Section 4, standardization is a very efficient method of integration, because it eliminates incompatibilities and makes direct cross-operations possible. Usually, in an NVO a standard middleware is deployed. All member enterprises may contact one another adapting their own computer management system just to the middleware standard.

If standards used in an NVO are widely accepted, standardization provides onlylimited risk to an enterprise aiming at joining the NVO. On the contrary, if the required standards are internal de-facto standards of the dominating partner within an NVO, the risk for a partner enterprise related with the acceptance of these standards is much higher, because internal de-facto standards create dependence. Also, standards accepted within an NVO are not necessarily optimal for activities outside the NVO performed by a member enterprise. Widely accepted standard solutions reduce costs, but do not provide competitive advantage. In general, standardization is a good method of integration for tightly coupled NVOs aiming at long lasting cooperation.

An alternative method of integration of computer management systems is intermediation by software agents. Intermediation is recommended for loosely coupled NVOs, cooperating only temporarily. Intermediation conserves autonomy of a partner enterprise and permits to gain competitive advantage following from its non-standard solutions.

A software agent is an autonomous program that independently performs given tasks at a given place and a given time according to the pre-programmed orders of its owner. In case of intermediation within an NVO, a software agent observes selected data of the partner enterprise, which reflect the current status of its business processes, and reports to the NVO management system all interesting events. Every partner enterprise may have a different software system and different data. For every partner enterprise an "interesting event" means something different. What is interesting, what is not, may be easily redefined in runtime. Every interesting event may happen in an arbitrary moment. Software agents permit extremely dynamic ad-hoc NVOs.

The second integration required by an NVO concerns representatives of partner enterprises who mutually cooperate via network to achieve NVO goals. They have to become a virtual team. It is relatively easy to provide the members of a virtual team with appropriate information, knowledge, and cooperation tools, which are mostly the same as in the case of computer supported collocated teams. These tools include: cooperation tools, project management tools, time management tools, document management tools, change management tools and tools to access to NVO databases and knowledgebases. Difficulties come from the fact that partner enterprises are by definition heterogeneous in order to achieve complementarities. While managing virtual teams, it is necessary to consider different business and national cultures, because they may determine important business factors.

The ultimate goal of a virtual team is common understanding of arising problems by all virtual team members to cooperatively provide a right solution. To correctly interpret information (about problems, opportunities, and solutions) knowledge is required. Knowledge is divided into: explicit knowledge (codified) and tacit knowledge (uncodified). To provide explicit knowledge, e-learning methods are used. 
Provision of common tacit knowledge is much more difficult, because tacit knowledge is acquired mostly by experience. To manage a virtual team, it is necessary to create a common business culture and to stimulate knowledge sharing. The measure of spread of tacit knowledge within a virtual team is the ability to solve problems, which may be tested in simulated environments.

\section{Ongoing Research}

Current research are focused on Virtual Breeding Environment, as an intermediary between a universe of enterprises and NVOs. The concept of Virtual Breeding Environment has been proposed within the framework of the ECOLEAD project as "an association of organizations and their related supporting institutions, adhering to a base long term cooperation agreement, and adoption of common operating principles and infrastructures, with the main goal of increasing their preparedness towards collaboration in potential Virtual Organizations" [2]. A very important research problem are methods aiming at facilitation of creation of Virtual Breeding Environments. Their creation requires strategic and management decision-making processes substantially different from those met in traditional organizations. Various aspects have to be addressed: from technological, organizational, and economic, to legislative, psychological, and cultural ones. Aspects related with business and knowledge has been already considered, while social aspects need to be more deeply investigated. In [11] social network analysis is applied to this end. Metrics are proposed as a way for a planner of a Virtual Breeding Environment to express social requirements.

In [9] methods are proposed that equip Virtual Breeding Environment with possibilities of NVO modeling aiming at NVO partners search and selection, definition of partner roles and finally NVO composition. A possibility of modeling an NVO before its composition and running is a very important factor of its success.

When an NVO is running, an important problem is its performance measurement. Basing on measurement results it is possible to early detect friction points, improve collaboration between partners, and adapt the NVO to business needs. However, measuring performance of a distributed organization as a whole is not an easy task. In [10] a Reference Model for NVO performance measurement is presented. This model comprises a set of common terms that are used to describe key performance indicators.

\section{Conclusions}

As follows from the above, NVOs provide a chance for SMEs to successfully play a significant role on global markets. It is, however, difficult for traditional SMEs to meet requirements of NVOs, which concern skills of SME employees and external integration of SME software systems.

To help Polish SMEs, a special priority axis has been included to the Operational Programme Innovative Economy financed by European Commission in 2007-2013 [7]. 460 million Euro has been allocated to this priority axis. An SME that obtains a grant from this priority axis may finance both adaptation of software systems and business process reengineering, aiming at cooperation with business partners via 
network. This programme brings hope of upgrading Polish SMEs to increase their potential of cooperation and as a consequence to extend markets on which they operate to the whole European Union, and beyond.

A social consequence of this program is creation of knowledge based workplaces related to international cooperation mostly for young, well-educated Polish people.

As this programme have just started, it is too early to report its detailed results.

\section{References}

1. Brunett, K., Fishman, G. (eds.): The NVO Way of Doing Business. The Bridge: Connecting Business and Technology Strategies. Cisco Systems Internet Business Solution Group (2003)

2. Camarinha-Matos, L.M., Afarmanesh, H., Galeano, N.: Characterization of Key Components, Features, and Operating Principles of the Virtual Breeding Environment. Deliverable 21.1. ECOLEAD (2004)

3. Camarinha-Matos, L.M., Afsarmanesh, H., Ollus, M. (eds.): Virtual Organizations: Systems and Practices. Springer, Heidelberg (2005)

4. Camarinha-Matos, L.M., Afsarmanesh, H., Ortiz, A. (eds.): Collaborative Networks and their Breeding Environments. Springer, Heidelberg (2005)

5. Cellary, W.: Globalization from the Information and Communication Perspective. In: Janowski, T., Mohanty, H. (eds.) ICDCIT 2007. LNCS, vol. 4882, pp. 283-292. Springer, Heidelberg (2007)

6. Cellary, W.: People and Software in a Knowledge-Based Economy. Computer 38(1), 114-116 (2005)

7. Innovative Economy Operational Programme 2007-2013, Polish Ministry of Regional Development (2007), http: / /www.mrr.gov.pl/

8. Oslo Manual, Guidelines for Collecting and Interpreting Innovation Data, OECD (Organization for Economic Co-Operation and Development) and Eurostat (Statistical Office of the European Communities) (2005)

9. Paszkiewicz, Z., Picard, W.: Modeling Virtual Organization Architecture with the Virtual Organization Breeding Methodology. In: 10th IFIP Working Conference on Virtual Enterprises PRO-VE 2009, Thessaloniki (Grece), October 7-9. Springer, Heidelberg (to appear, 2009)

10. Paszkiewicz, Z., Picard, W.: Reference Model for Performance Management in ServiceOriented Virtual Organization Breeding Environments. In: 10th IFIP Working Conference on Virtual Enterprises PRO-VE 2009, Thessaloniki (Grece), October 7-9. Springer, Heidelberg (to appear, 2009)

11. Świerzowicz, J., Picard, W.: Social Requirements for Virtual Organization Breeding Environments. In: 10th IFIP Working Conference on Virtual Enterprises PRO-VE 2009, Thessaloniki (Grece), October 7-9. Springer, Heidelberg (to appear, 2009) 